\title{
Applications of the Fractional Fourier Transform in Optics and Signal Processing - a Review
}

\author{
Haldun M. Ozaktas \\ Bilkent University, Dep. of Electrical Engineering, 06533 Bilkent, Ankara, TURKEY \\ David Mendlovic \\ Tel-Aviv University, Fac. of Engineering, 69978 Tel-Aviv, ISRAEL
}

The fractional Fourier transform The fractional Fourier transform is a generalization of the common Fourier transform with an order parameter $a$. Mathematically, the $a$ th order fractional Fourier transform is the $a$ th power of the fractional Fourier transform operator. The $a=1$ st order fractional transform is the common Fourier transform. The $a=0$ th transform is the function itself. With the development of the fractional Fourier transform and related concepts, we see that the common frequency domain is merely a special case of a continuum of fractional domains, and arrive at a richer and more general theory of alternate signal representations, all of which are elegantly related to the notion of space-frequency distributions. Every property and application of the common Fourier transform becomes a special case of that for the fractional transform. In every area in which Fourier transforms and frequency domain concepts are used, there exists the potential for generalization and improvement by using the fractional transform. $[1,2,3,4,5,6,7,8,9,10,11,12,13,14,15,16,17,18,19,20,21,22,23,24,25]$

For instance, the theory of optimal Wiener filtering in the common Fourier domain can be generalized to optimal filtering in fractional domains, resulting in smaller mean square errors at practically no additional cost [27, 28]. The well-known result stating that the far-field diffraction pattern of an aperture is in the form of the Fourier transform of the aperture can be generalized to state that at closer distances, one observes the fractional Fourier transform of the aperture $[40,41,42,43]$.

Applications The fractional Fourier transform has been found to have several applications in analogue optical information processing, or Fourier optics. This transform allows a reformulation of this area in a much more general way than the standard formulation. It has also allowed a generalization of the Fourier transform and the notion of the frequency domain, which are very central concepts in signal processing, and is expected to have an impact in the form of deeper understanding or new applications in every area in which the Fourier transform plays a significant role.

Signal processing Some applications which have already been investigated or suggested include time- or space-variant filtering and signal detection $[9,26,27,28,29,30]$, time- or space-variant multiplexing and data compression [9], correlation, matched filtering, and pattern recognition [31, 32], study of time- or space-frequency distributions [7, 9, 10,12, 14, 22, 33], signal synthesis [34], radar [28], phase retrieval [65, 68], and solution of differential equations $[2,3]$. We believe that these represent only a fraction of the possible applications.

The relationship to wavelet transforms and neural networks has been pointed out in $[9,35]$ and other fractional transformations have been explored in $[36,37]$. The discrete-time fractional Fourier transform and its digital computation are investigated in [38, 39].

Optical propagation and diffraction, and Fourier optics It has been shown that there exists a fractional Fourier transform relation between the (appropriately scaled) optical amplitude distributions on two spherical reference surfaces with given radii and separation. This result provides an alternative statement of the law of propagation and allows us to pose the fractional Fourier transform as a tool for analyzing and describing a rather general class of optical systems. One of the central results of diffraction theory is that the far-field diffraction pattern is the Fourier transform of the diffracting object. It is possible to generalize this result by showing that the field patterns at closer distances are the fractional Fourier transforms of the diffracting object. [40, 41, 42, 43, 44, 45, 46, 47, 48]

More generally, in an optical system involving many lenses separated by arbitrary distances, it is possible to show that the amplitude distribution is continuously fractional Fourier transformed as it propagates through the system. The order $a(z)$ of the fractional transform observed at the distance $z$ along the optical axis is a continuous monotonic 
increasing function. As light propagates, its distribution evolves through fractional transforms of increasing orders. Wherever the order of the transform $a(z)$ is equal to $4 j+1$ for any integer $j$, we observe the Fourier transform of the input. Wherever the order is equal to $4 j+2$, we observe an inverted image, etc. [40].

Propagation in graded-index media, and Gaussian beam propagation can also be studied in terms of the fractional Fourier transform $[4,5,6,41,49]$.

Optical signal processing The fractional Fourier transform can be optically realized in a similar manner as the common Fourier transform. The fact that the fractional Fourier transform can be realized optically means that the many applications of the transform in signal processing can also be carried over to optical signal processing. $[4,5,6,7,29,40,42,43,45,46,47,50,51,52,53,54,55,56,57,58,59,60,61,62]$

Other optical applications These include spherical mirror resonators (lasers) [41], optical systems and lens design [63], quantum optics [64, 65, 66, 67], phase retrieval [65, 68, 69], statistical optics [70], beam shaping [71, 72], and Legendre transformations [73].

\section{References}

[1] Status report on The Fractional Fourier Transform. A. W. Lohmann, D. Mendlovic, and Z. Zalevsky, eds. Tel-Aviv University, Faculty of Engineering, Tel-Aviv, Israel, 1995.

[2] A. C. McBride and F. H. Kerr. On Namias's fractional Fourier transform. IMA J. Appl. Math., 39:159-175, 1987.

[3] V. Namias. The fractional order Fourier transform and its application to quantum mechanics. J. Inst. Maths. Applics., 25:241-265, 1980.

[4] H. M. Ozaktas and D. Mendlovic. Fourier transforms of fractional order and their optical interpretation. Opt. Commun., 101:163-169, 1993.

[5] D. Mendlovic and H. M. Ozaktas. Fractional Fourier transformations and their optical implementation, I. J. Opt. Soc. Am. A, 10:1875-1881, 1993.

[6] H. M. Ozaktas and D. Mendlovic. Fractional Fourier transformations and their optical implementation, II. J. Opt. Soc. Am. A, 10:2522-2531, 1993.

[7] A. W. Lohmann. Image rotation, Wigner rotation, and the fractional order Fourier transform. J. Opt. Soc. Am. A, 10:2181-2186, 1993

[8] D. Mendlovic, H. M. Ozaktas, and A. W. Lohmann. Graded-index fibers, Wigner-distribution functions, and the fractional Fourier transform. Appl. Opt., 33:6188-6193, 1994.

[9] H. M. Ozaktas, B. Barshan, D. Mendlovic, and L. Onural. Convolution, filtering, and multiplexing in fractional Fourier domains and their relation to chirp and wavelet transforms. J. Opt. Soc. Am. A, 11:547-559, 1994.

[10] L. B. Almeida. The fractional Fourier transform and time-frequency representations. IEEE Trans. Signal Processing, 42:3084-3091, 1994.

[11] J. C. Wood and D. T. Barry. Radon transformation of time-frequency distributions for analysis of multicomponent signals. IEEE Trans. Signal Processing, 42:3166-3177, 1994.

[12] A. W. Lohmann and B. H. Soffer. Relationships between the Radon-Wigner and fractional Fourier transforms. J. Opt. Soc. Am. A, 11:1798-1801, 1994.

[13] H. M. Ozaktas and O. Aytür. Fractional Fourier domains. Signal Processing, 46:119-124, 1995.

[14] H. M. Ozaktas, N. Erkaya, and M. A. Kutay. Effect of fractional Fourier transformation on time-frequency distributions belonging to the Cohen class. To appear in IEEE Signal Processing Lett.

[15] K. B. Wolf. Construction and properties of canonical transforms. In Integral Transforms in Science and Engineering, Plenum Press, New York, 1979, chapter 9.

[16] O. Seger. Model Building and Restoration with Applications in Confocal Microscopy, Ph.D. thesis, Linköping University, Sweden, 1993.

[17] D. Mendlovic, H. M. Ozaktas, and A. W. Lohmann. Self Fourier functions and fractional Fourier transforms. Opt. Commun., 105:36-38, 1994.

[18] D. Mustard. Uncertainty principles invariant under the fractional Fourier transform. J. Austral. Math. Soc. B, 33:180-191, 1991. 
[19] G. S. Agarwal and R. Simon. A simple realization of fractional Fourier transforms and relation to harmonic oscillator Green's function. Opt. Commun., 110:23-26, 1994.

[20] Y. B. Karasik. Expression of the kernel of a fractional Fourier transform in elementary functions. Opt. Lett., 19:769-770, 1994.

[21] C.-C. Shih. Fractionalization of Fourier transform. Opt. Commun., 118:495-498, 1995.

[22] D. Mendlovic, Z. Zalevsky, R. G. Dorsch, Y. Bitran, A. W. Lohmann, and H. M. Ozaktas. A new signal representation based on the fractional Fourier transform: definitions. J. Opt. Soc. Am. A, 12:2424-2431, 1995.

[23] S. Abe and J. T. Sheridan. Optical operations on wave functions as the Abelian subgroups of the special affine Fourier transformation. Opt. Lett., 19:1801-1803, 1994

[24] S. Abe and J. T. Sheridan. Generalization of the fractional Fourier transformation to an arbitrary linear lossless transformation: an operator approach. J. Phys. A, 27:4179-4187, 1994. Corrigenda in 7937-7938.

[25] S. Abe and J. T. Sheridan. Almost-Fourier and almost-Fresnel transformations. Opt. Commun., 113:385-388, 1995.

[26] H. M. Ozaktas, B. Barshan, and D. Mendlovic. Convolution and Filtering in Fractional Fourier Domains. Optical Review, 1:15-16, 1994.

[27] M. A. Kutay, H. M. Ozaktas, L. Onural, and O. Artkan. Optimal filtering in fractional Fourier domains. In Proc. 1995 Int. Conf. Acoustics, Speech, and Signal Processing, IEEE, Piscataway, New Jersey, 1995, pages $937-940$.

[28] M. A. Kutay, H. M. Ozaktas, O. Arkan, and L. Onural. Optimal Filtering in Fractional Fourier Domains. Submitted to IEEE Trans. Signal Processing.

[29] D. Mendlovic, Z. Zalevsky, A. W. Lohmann, and R. G. Dorsch. Signal spatial-filtering using the localized fractional Fourier transform. To appear in Opt. Commun.

[30] J. C. Wood and D. T. Barry. Tomographic time-frequency analysis and its application toward time-varying filtering and adaptive kernel design for multicomponent linear-FM signals. IEEE Trans. Signal Processing, 42:2094$2104,1994$.

[31] D. Mendlovic, H. M. Ozaktas, and A. W. Lohmann. Fractional correlation. Appl. Opt., 34:303-309, 1995.

[32] Y. Bitran, Z. Zalevsky, D. Mendlovic, and R. G. Dorsch. Performance analysis of the fractional correlation operation. To appear in Appl. Opt.

[33] J. R. Fonollosa and C. L. Nikias. A new positive time-frequency distribution. In Proc. 1994 Int. Conf. Acoustics, Speech, and Signal Processing, IEEE, Piscataway, New Jersey, 1994, pages IV-301-IV-304.

[34] J. C. Wood and D. T. Barry. Linear signal synthesis using the Radon-Wigner transform. IEEE Trans. Signal Processing, 42:2105-2111, 1994.

[35] S.-Y. Lee and H. H. Szu. Fractional Fourier transforms, wavelet transforms, and adaptive neural networks. Opt. Eng., 33:2326-2330, 1994.

[36] A. W. Lohmann, D. Mendlovic, Z. Zalevsky, and R. G. Dorsch. Some important fractional transformations for signal processing. To appear in Opt. Commun.

[37] A. W. Lohmann, D. Mendlovic, and Z. Zalevsky. Fractional Hilbert transform. To appear in Opt. Lett.

[38] H. M. Ozaktas, O. Arikan, M. A. Kutay, and G. Bozdağı. Digital computation of the fractional Fourier transform. To appear in IEEE Trans. Signal Processing.

[39] B. Santhanam and J. H. McClellan. The DRFT-a rotation in time-frequency space. In Proc. 1995 Int. Conf. Acoustics, Speech, and Signal Processing, IEEE, Piscataway, New Jersey, 1995, pages 921-924.

[40] H. M. Ozaktas and D. Mendlovic. Fractional Fourier optics. J. Opt. Soc. Am. A, 12:743-751, 1995.

[41] H. M. Ozaktas and D. Mendlovic. Fractional Fourier transform as a tool for analyzing beam propagation and spherical mirror resonators. Opt. Lett., 19:1678-1680, 1994.

[42] P. Pellat-Finet. Fresnel diffraction and the fractional-order Fourier transform. Opt. Lett., 19:1388-1390, 1994.

[43] P. Pellat-Finet and G. Bonnet. Fractional order Fourier transform and Fourier optics. Opt. Commun., 111:141$154,1994$.

[44] H. M. Ozaktas. Every Fourier optical system is equivalent to consecutive fractional Fourier domain filtering. To appear in Appl. Opt.

[45] L. M. Bernardo and O. D. D. Soares. Fractional Fourier transforms and imaging. J. Opt. Soc. Am. A, 11:26222626, 1994. 
[46] L. M. Bernardo and O. D. D. Soares. Fractional Fourier transforms and optical systems. Opt. Commun., 110:517$5 \sim 2,1994$

[47] T. Alieva, V. Lopez, F. Agullo-Lopez, and L. B. Almeida. The fractional Fourier transform in optical propagation problems. J. Mod. Opt., 41:1037-1044, 1994.

[48] M. E. Marhic. Roots of the identity operator and optics. J. Opt. Soc. Am. A, 12:1448-1459, 1995.

[49] T. Alieva and F. Agulló-López. Reconstruction of the optical correlation function in a quadratic refractive index medium. Opt. Commun., 114:161-169, 1995. Erratum in 118:657, 1995.

[50] Y. Bitran, D. Mendlovic, R. G. Dorsch, A. W. Lohmann, and H. M. Ozaktas. Fractional Fourier transform: simulations and experimental results. Appl. Opt., 34:1329-1332, 1995.

[51] S. Liu, J. Xu, Y. Zhang, L. Chen, and C. Li. General optical implementation of fractional Fourier transforms. Opt. Lett., 20:1053-1055, 1995.

[52] S. Liu, J. Wu, and C. Li. Cascading the multiple stages of optical fractional Fourier transforms under different variable scales. Opt. Lett., 20:1415-1417, 1995.

[53] D. Mendlovic, Z. Zalevsky, N. Konforti, R. G. Dorsch, and A. W. Lohmann. Incoherent fractional Fourier transform and its optical implementation. Appl. Opt., 34:7615-7620, 1995.

[54] R. G. Dorsch. Fractional Fourier transformer of variable order based on a modular lens system. Appl. Opt., 34:6016-6020, 1995.

[55] A. W. Lohmann. A fake zoom lens for fractional Fourier experiments. Opt. Commun., 115:437-443, 1995.

[56] D. Mendlovic, Y. Bitran, C. Ferreira, J. Garcia, and H. M. Ozaktas. Anamorphic fractional Fourier transforming-optical implementation and applications. Appl. Opt., 34:7451-7456, 1995.

[57] A. Sahin, H. M. Ozaktas, and D. Mendlovic. Optical implementation of the two-dimensional fractional Fourier transform with different orders in the two dimensions. Opt. Commun., 120:134-138, 1995.

[58] C.-C. Shih. Optical interpretation of a complex-order Fourier transform. Opt. Lett., 20:1178-1180, 1995.

[59] R. G. Dorsch, A. W. Lohmann, Y. Bitran, D. Mendlovic, and H. M. Ozaktas. Chirp filtering in the fractional Fourier domain. Appl. Opt., 33:7599-7602, 1994.

[60] S. Granieri, O. Trabocchi, and E. E. Sicre. Fractional Fourier transform applied to spatial filtering in the Fresnel domain. Opt. Commun., 119:275-278, 1995.

[61] D. Mendlovic, Y. Bitran, R. G. Dorsch, and A. W. Lohmann. Optical fractional correlation: experimental results. Appl. Opt., 12:1665-1670, 1995.

[62] A. W. Lohmann and D. Mendlovic. Fractional Fourier transform: photonic implementation. Appl. Opt., 33:7661$7664,1994$.

[63] R. G. Dorsch and A. W. Lohmann. Fractional Fourier transform used for a lens design problem. Appl. Opt., 34:4111-4112, 1995.

[64] O. Aytür and H. M. Ozaktas. Non-orthogonal domains in phase space of quantum optics and their relation to fractional Fourier transforms. Opt. Commun., 120:166-170, 1995.

[65] M. G. Raymer, M. Beck, and D. F. McAlister. Complex wave-field reconstruction using phase-space tomography. Phys. Rev. Lett., 72:1137-1140, 1994.

[66] D. T. Smithey, M. Beck, M. G. Raymer, and A. Faridani. Measurement of the Wigner distribution and the density matrix of a light mode using optical homodyne tomography: application to squeezed states and the vacuum. Phys. Rev. Lett., 70:1244-1247, 1993.

[67] B. Yurke, W. Schleich, and D. F. Walls. Quantum superpositions generated by quantum nondemolition measurements. Phys. Rev. A, 42:1703-1711, 1990.

[68] D. F. McAlister, M. Beck, L. Clarke, A. Meyer, and M. G. Raymer. Optical phase-retrieval by phase-space tomography and fractional-order Fourier transforms. Opt. Lett., 20:1181-1183, 1995.

[69] M. Beck, M. G. Raymer, I. A. Walmsley, and V. Kong. Chronocyclic tomography for measuring the amplitude and phase structure of optical pulses. Opt. Lett., 18:2041-2043, 1993.

[70] M. F. Erden, H. M. Ozaktas, and D. Mendlovic. Propagation of mutual intensity expressed in terms of the fractional Fourier transform. To appear in J. Opt. Soc. Am. A.

[71] Z. Zalevsky, D. Mendlovic, and R. G. Dorsch. The Gerchberg-Saxton algorithm applied in the fractional Fourier or the Fresnel domains. To appear in Opt. Lett.

[72] M. F. Erden, H. M. Ozaktas, and D. Mendlovic. Synthesis of mutual intensity distributions using the fractional Fourier transform. To appear in Opt. Commun.

[73] M. A. Alonso and G. W. Forbes. Fractional Legendre transformation, To appear in J. Phys. A. 